\title{
Non-inertial quantum mechanical fluctuations
}

\author{
Haret Rosu \\ Guanajuato University \\ Leon \\ Guanajuato \\ Mexico \\ E-mail: rosu@ifug3.ugto.mx
}

\begin{abstract}
Zero point quantum fluctuations as seen from non-inertial reference frames are of interest for several reasons. In particular, because phenomena such as Unruh radiation (acceleration radiation) and Hawking radiation (quantum leakage from a black hole) depend intrinsically on both quantum zero-point fluctuations and some appropriate notion of an accelerating vacuum state, any experimental test of zero-point fluctuations in non-inertial frames is implicitly a test of the foundations of quantum field theory, and the Unruh and Hawking effects.
\end{abstract}

To appear as a chapter in the book Artificial Black Holes (World Scientific) edited by M. Novello, M. Visser, and G. Volovik. 


\subsection{Introduction}

Analog or not, the ultimate goal of the physics described in this book is to find clear evidence for gravitational and non-inertial vacuum radiation. Recognized as some of the most important paradigms of present-day theoretical physics, the Hawking and Unruh effects are (as yet) not much more than academic results which, because of the scales of the required energies/ accelerations/ masses, are not easy to implement in real laboratory experiments. Indeed, from the experimental standpoint, they might seem to be merely exotic interpretations for what could be explained by far more mundane physical effects in quantum electrodynamics, quantum optics, and hydrodynamics.

As counterpoint, in the case of the Unruh effect the so-called detector method provides a well-defined radiation pattern that may be thought of as vacuum noise, and should be kept under consideration for possible experimental detection in clean analog experiments. In this chapter, old results of Letaw on scalar vacuum radiation patterns are used to emphasize the radiometric nature of the various Frenet-Serret invariants for certain classes of "stationary" worldlines. This formalism is an extension of, and alternative to, the usual notion: that of adopting the thermal interpretation of the vacuum excitations as seen by a uniformly accelerated quantum detector (Unruh's interpretation).

I focus next on the electromagnetic vacuum noise, surveying the HacyanSarmiento approach for calculating physical quantities in the electromagnetic vacuum. The application of this approach to circular worldlines led Mane to propose the identification of Hacyan-Sarmiento zero-point radiation with the ordinary synchrotron radiation; but here I provide some simple counter-arguments. I also briefly discuss Bell and Leinaas' proposal of considering electrons in storage rings as prototypes for an Unruh-DeWitt spin polarization detector. In the final section, I sketch the similarity between the Unruh effect and the so-called "anomalous Doppler effect". A separate observation of the latter would mean a confirmation of the possibility of the first.

\subsection{Vacuum Field Noise — VFN}

The quantum vacuum field noise (VFN) [1] that is recorded by a detector moving along some classical trajectory will in general depend on that trajectory. For certain restricted classes of worldline trajectories $x^{\mu}(s)$, most usefully parametrized in terms of proper time $s$, the observed power spectrum is stationary (timeindependent). Experimental observation of the power spectrum of the vacuum field noise is then an important diagnostic tool that can be inverted to extract information about the form of the trajectory - specifically, the curvature invariants (Frenet-Serret invariants) of the worldline. As usual, I model an idealized detector as a simple two-level quantum system [usually known as an UnruhDeWitt detector]. For scalar quantum field vacua there are six broad classes of trajectory that lead to stationary noise spectra. Basic results were derived by Letaw [2] some time ago, and are reviewed below. They might be of direct ex- 
perimental interest in the acoustic analogy. On the other hand, one should also keep in mind that non-stationary vacuum noises are not completely beyond experimental reach, and can be analyzed by related mathematical methods which I briefly comment on.

\subsubsection{The detector method in quantum field theory}

For the idealized Unruh-DeWitt detector, the interaction between the detector [endowed with a monopole moment $Q(s)$ ] and the scalar field $\phi(s)$ is described by

$$
L_{\text {int }}=\lambda Q(s) \phi(s),
$$

where $\phi(s)=\phi(x(s))$ is the field along the worldline of the detector and $\lambda$ is a small coupling constant that I re-scale to $\lambda=1$ (since it is not important for current considerations). Detecting particles in the Unruh-DeWitt apparatus requires one to adopt adiabatic switching appropriate for the perturbative approach. At $s=-\infty$, the detector is in the ground-state $\left|E_{0}\right\rangle$ and the field is in the Minkowski vacuum $\left|0_{M}\right\rangle$. After the detector-field interaction is switched on, the detector would not remain in the state $\left|E_{0}\right\rangle$, but would make a transition to $\left|E_{1}\right\rangle$. It is said that the detector "detects" some particles. Then, the transition amplitude for the detector field system to be found in $\left|E_{1}, \psi\right\rangle$ at $s=+\infty$ is given by first-order perturbation theory as

$$
A=i\left\langle E_{1}, \psi\left|\int_{-\infty}^{\infty} \mathrm{d} s Q(s) \phi(s)\right| E_{0}, 0_{M}\right\rangle .
$$

In order for first-order perturbation theory to apply one has to assume that the matrix element of $Q$ is sufficiently small. On the other hand, from the time evolution of the operator $Q$ in the Heisenberg picture

$$
Q(s)=e^{i H_{D} s / \hbar} Q(0) e^{-i H_{D} s / \hbar},
$$

where $H_{D}$ is the detector Hamiltonian, one immediately obtains

$$
A=i\left\langle E_{1}|Q(0)| E_{0}\right\rangle \int_{-\infty}^{\infty} \mathrm{d} s e^{i\left(E_{1}-E_{0}\right) s / \hbar}\left\langle\psi|\phi(s)| 0_{M}\right\rangle .
$$

After summation over all final states of the field $|\psi\rangle$, the transition rate, i.e., the transition probability per unit proper time from $E_{0}$ to $E_{1}$ is

$$
\frac{\mathrm{d} P}{\mathrm{~d} s}=\left|\left\langle E_{1}|Q(0)| E_{0}\right\rangle\right|^{2} S(\omega),
$$

where $\omega=\left(E_{1}-E_{0}\right) / \hbar$ and

$$
S(\omega)=\int_{-\infty}^{\infty} \mathrm{d}\left(s-s^{\prime}\right) e^{-i \omega\left(s-s^{\prime}\right)} g\left(s-s^{\prime}\right) .
$$

The integrand $g$ is the Minkowski vacuum expectation value of the autocorrelation function (the Wightman function)

$$
g\left(s-s^{\prime}\right)=\left\langle 0_{M}\left|\phi(s) \phi\left(s^{\prime}\right)\right| 0_{M}\right\rangle .
$$


Thus, $S$ looks like a response function (or power spectrum) and $g$ as the "quantum noise" in the Minkowski vacuum along the worldline $x(s)$. The peculiar feature of this argument is that the quantum detector performs an "up" transition and at the same time sees ('emits') a 'radiation' spectrum. From the phenomenological point of view such a situation can also be encountered in the case of the anomalous Doppler effect (ADE) as has been remarked by Frolov and Ginzburg [3] (see Section 0.4 below).

\subsubsection{Six types of stationary scalar VFN}

In general, the scalar quantum field vacuum does not possess a stationary vacuum excitation spectrum (abbreviated as SVES) for all types of classical relativistic trajectories on which the Unruh-DeWitt detector could move. Nevertheless, linear uniform acceleration is not the only case with that property. This was shown by Letaw, who extended Unruh's considerations, obtaining six broad classes of worldlines with SVES for an Unruh-DeWitt monopole detector (SVES-1 to SVES-6, see below). The line of argument is the following: The Unruh-DeWitt detector is effectively immersed in a scalar bath of vacuum fluctuations. Its rate of excitation is determined by the energy spectrum of the scalar bath, which can be expressed as the density of states times a cosine-Fourier transform of the Wightman correlation function of the scalar field. Since the Wightman function is directly expressed in terms of the inverse of the geodesic interval what one needs to calculate is a Fourier transform of the inverse of the geodesic interval $\int \mathrm{d} s=\int \sqrt{\mathrm{d} x_{\mu}^{2}}$. Moreover, stationarity means that the Wightman function depends only on the proper time interval.

As shown by Letaw, the stationary worldlines are solutions of some generalized Frenet-Serret (FS) equations on which the condition of constant curvature invariants is imposed. That is, one is interested in worldlines of constant curvature $\kappa$, torsion $\tau$, and hyper-torsion (bi-torsion) $\nu$, respectively. These curvature invariants can be easily built from the tangent, normal, and binormal vectors and their derivatives. They have physical interpretation in terms of the observer's acceleration and angular velocities. Notice that one can employ other frameworks, such as the Newman-Penrose spinor formalism as recently invoked by Unruh [ [], but the Frenet-Serret framework is in overwhelming use throughout physics. It is worth remarking that before Letaw, the Frenet-Serret invariants have been discussed by Honig et al [5] in their study of the motion of charged particles in homogeneous electromagnetic fields. Honig et al discovered an interesting connection with the two Lorentz invariants of the electromagnetic field, $E^{2}-H^{2}$ and $\vec{E} \cdot \vec{H}$ :

$$
\begin{gathered}
E^{2}-H^{2} \propto \kappa^{2}-\tau^{2}-\nu^{2}, \\
\vec{E} \cdot \vec{H} \propto \kappa \nu .
\end{gathered}
$$

It is amusing to note that chirality (handedness) in the electromagnetic sense $(\vec{E} \cdot \vec{H} \neq 0)$ is proportional to chirality in the worldline sense (nonzero hypertorsion; $\nu \neq 0$.) 
The six stationary scalar VFN can be classified according to the curvature scalars of the corresponding worldlines:

1. $\underline{\kappa=\tau=\nu=0} \rightarrow$ inertial, uncurved worldlines (constant velocity).

SVES-1 is a trivial cubic spectrum

$$
S_{1}(E)=\frac{E^{3}}{4 \pi^{2}} .
$$

This can be interpreted as a vacuum state of zero point energy $E / 2$ per mode, with density of states $E^{2} / 2 \pi^{2}$.

2. $\kappa \neq 0, \tau=\nu=0 \rightarrow$ hyperbolic worldlines (constant rectilinear acceleration).

SVES-2 is Planckian allowing the interpretation of $\kappa / 2 \pi$ as 'thermodynamic' temperature. In the dimensionless variable $\epsilon_{\kappa}=E / \kappa$ the vacuum spectrum reads

$$
S_{2}\left(\epsilon_{\kappa}\right)=\frac{\epsilon_{\kappa}^{3}}{2 \pi^{2}\left(e^{2 \pi \epsilon_{\kappa}}-1\right)} .
$$

The physically observed spectrum would be a linear combination of SVES1 and SVES-2.

3. $|\kappa|<|\tau|, \nu=0, \rho^{2}=\tau^{2}-\kappa^{2} \rightarrow$ helical worldlines.

SVES-3 is a complicated analytic function corresponding to the case 4 below only in the limit $\kappa \gg \rho$

$$
S_{3}\left(\epsilon_{\rho}\right) \stackrel{\kappa / \rho \rightarrow \infty}{\longrightarrow} S_{4}\left(\epsilon_{\kappa}\right) .
$$

Letaw plotted the numerical integral $S_{3}\left(\epsilon_{\rho}\right)$, where $\epsilon_{\rho}=E / \rho$, for various values of $\kappa / \rho$.

4. $\kappa=\tau, \nu=0 \rightarrow$ the spatially projected worldlines are the "semicubical $\overline{\text { parabolas", } y} \propto \kappa x^{3 / 2}$, containing a cusp (at $x=0$ ) where the direction of motion is reversed.

SVES-4 is analytic, and since there are two equal curvature invariants $(\kappa=\tau)$ one can use the dimensionless energy variable $\epsilon_{\kappa}=E / \kappa$

$$
S_{4}\left(\epsilon_{\kappa}\right)=\frac{\epsilon_{\kappa}^{2}}{8 \pi^{2} \sqrt{3}} e^{-2 \sqrt{3} \epsilon_{\kappa}} .
$$

It is worth noting that $S_{4}$, being a monomial times an exponential, is rather close to the Wien spectrum $S_{W} \propto \epsilon^{3} e^{- \text {const. } \epsilon}$. 
5. $|\kappa|>|\tau|, \nu=0, \sigma^{2}=\kappa^{2}-\tau^{2} \rightarrow$ the spatially projected worldlines are catenaries, curves of the type $x=\kappa \cosh (y / \tau)$.

In general, SVES-5 cannot be found analytically. It is an intermediate case, which for $\tau / \sigma \rightarrow 0$ tends to SVES-2, whereas for $\tau / \sigma \rightarrow \infty$ tends toward SVES-4

$$
S_{2}\left(\epsilon_{\kappa}\right) \stackrel{0 \longleftarrow \tau / \sigma}{\longleftarrow} S_{5}\left(\epsilon_{\sigma}\right) \stackrel{\tau / \sigma \rightarrow \infty}{\longrightarrow} S_{4}\left(\epsilon_{\kappa}\right) .
$$

6. $\nu \neq 0, \kappa$ and $\tau$ arbitrary $\rightarrow$ rotating worldlines uniformly accelerated normal to their plane of rotation.

SVES-6 forms a three-parameter set of curves. The corresponding trajectories are a superposition of the constant linearly accelerated motion and uniform circular motion. SVES-6 has not been calculated by Letaw, not even numerically.

Thus, only the hyperbolic worldlines, having just one nonzero curvature invariant, allow for a Planckian SVES. Only that case allows for a strictly oneto-one mapping between the curvature invariant $\kappa$ and the 'thermodynamic' temperature in the celebrated form $T_{U}=\kappa / 2 \pi$. The vacuum field noise of semicubical parabolas can be fitted by a Wien-type spectrum, the radiometric parameter then corresponding to both curvature and torsion. The other stationary cases, being nonanalytic, lead to the approximate determination of the curvature invariants defining locally the classical worldline on which the relativistic quantum detector moves.

One very general and important statement regarding the universal nature of the kinematical Frenet-Serret parameters occurring in various important quantum field model problems can be formulated as follows:

There exist accelerating classical trajectories (worldlines) on which moving ideal (two-level) quantum systems can detect the scalar vacuum environment as a stationary quantum field vacuum noise with a spectrum directly related to the curvature invariants of the worldline, thus allowing for a radiometric interpretation of those invariants.

According to these results, it seems more appropriate to replace the thermal interpretation of Unruh by the radiometric interpretation of the Frenet-Serret invariants. The latter is more general and describes in a more precise way the physical situation to which the Unruh effect refers, that of a quantum particle moving along a classical relativistic trajectory. 


\subsubsection{Explicit formulae for the spectra}

One can calculate the spectrum of vacuum field noise by means of the following general formula

$$
S_{j}(E)=\left|\frac{E^{2}}{4 \pi^{3}} \int_{-\infty}^{\infty} \frac{e^{-i E s} \mathrm{~d} s}{\left[x_{\mu}(s)-x_{\mu}(0)\right]\left[x^{\mu}(s)-x^{\mu}(0)\right]}\right|=\frac{E^{2}}{4 \pi^{3}}\left|\mathrm{I}_{j}\right|, \quad j=1 \ldots 6,
$$

where $x^{\mu}(s)$ is an arbitrary point on the worldline and $x^{\mu}(0)$ is the initial point. The signature of the Minkowski metric is $\eta_{\mu \nu}=(1,-1,-1,-1)$. I confirm Letaw's results by sketching the calculation of the integrals $\mathrm{I}_{j}$ for the six stationary cases. Simple details that have been skipped by Letaw can be found here.

1. The recta. The worldline is $x^{\mu}(s)=(s, 0,0,0)$; with initial condition $x^{\mu}(0)=(0,0,0,0)$. The integral is

$$
\mathrm{I}_{1}=\int_{-\infty}^{+\infty} \frac{e^{-i E s}}{s^{2}} \mathrm{~d} s
$$

It can be evaluated by using Cauchy's residue theorem plus the expansion $e^{-i E s}=(1-i E s+\ldots)$. The value of the integral is $\pi i(-i E)=\pi E$, and therefore one gets the cubic spectrum. This inertial zero-point cubic spectrum will appear in all the other five stationary spectra as an additive background and therefore one may take into account only the non-cubic contributions as a measure of non-inertial vacuum effects.

2. The hyperbola. The worldline is $x^{\mu}(s)=\kappa^{-1}(\sinh \kappa s, \cosh \kappa s, 0,0)$; with the initial condition $x^{\mu}(0)=\kappa^{-1}(0,1,0,0)$. Now the integral is

$$
\mathrm{I}_{2}=\kappa \int_{-\infty}^{+\infty} \frac{e^{-i \epsilon_{\kappa} u}}{2(\cosh u-1)} \mathrm{d} u
$$

Writing $e^{-i \epsilon_{\kappa} u}=\cos \epsilon_{\kappa} u-i \sin \epsilon_{\kappa} u$, one makes use of formula 3.983.3 at page 505 in the fourth edition of the Table of Gradshteyn and Ryzhik (GR) to get

$$
\int_{0}^{+\infty} \frac{\cos (a x)}{\cosh x-1} d x=-\pi a \operatorname{coth}(\pi a)
$$

The sine integral can be evaluated using Cauchy's theorem

$$
-i \int_{-\infty}^{+\infty} \frac{\sin \left(\epsilon_{\kappa} x\right) d x}{\cosh x-1}=\pi \epsilon_{\kappa}
$$

Thus

$$
\begin{aligned}
\mathrm{I}_{2} & =-\pi \epsilon_{\kappa} \operatorname{coth}\left(\pi \epsilon_{\kappa}\right)+\frac{\pi \epsilon_{\kappa}}{2} \\
& =\pi \epsilon_{\kappa}\left[1-\operatorname{coth}\left(\pi \epsilon_{\kappa}\right)\right]-\frac{\pi \epsilon_{\kappa}}{2} \\
& =-2 \pi \epsilon_{\kappa} \frac{1}{e^{2 \pi \epsilon_{\kappa}}-1}-\frac{\pi \epsilon_{\kappa}}{2}
\end{aligned}
$$


where the first term leads to the Planckian spectrum and the latter to the cubic zero-point contribution.

3. The helix. The worldline is $x^{\mu}(s)=\rho^{-2}(\tau \rho s, \kappa \cos \rho s, \kappa \sin \rho s, 0)$; with initial condition $x^{\mu}(0)=\rho^{-2}(0, \kappa, 0,0)$. The integral reads

$$
\mathrm{I}_{3}=\rho \int_{-\infty}^{+\infty} \frac{e^{-i \epsilon_{\rho} u}}{2 \frac{\kappa^{2}}{\rho^{2}}(\cos u-1)+\frac{\tau^{2}}{\rho^{2}} u^{2}} \mathrm{~d} u
$$

where

$$
\frac{\tau^{2}}{\rho^{2}}-\frac{\kappa^{2}}{\rho^{2}}=1
$$

According to Letaw this integral is non-analytic and indeed I was not able to find any helpful formula in the GR Table.

\section{The semicubical parabola.}

The worldline is $x^{\mu}(s)=\left(s+\frac{1}{6} \kappa^{2} s^{3}, \frac{1}{2} \kappa s^{2}, \frac{1}{6} \kappa^{2} s^{3}, 0\right)$; with initial condition $x^{\mu}(0)=(0,0,0,0)$. The integral reads

$$
\mathrm{I}_{4}=\kappa \int_{-\infty}^{+\infty} \frac{e^{-i \epsilon_{\kappa} u} \mathrm{~d} u}{u^{2}\left(1+\frac{1}{12} u^{2}\right)}=\kappa \mathrm{I}_{1}-\kappa \int_{-\infty}^{+\infty} \frac{e^{-i \epsilon_{\kappa} u} \mathrm{~d} u}{\left(12+u^{2}\right)}
$$

Of interest is only the second integral that can be found in the GR Table at page 359

$$
\int_{-\infty}^{+\infty} \frac{e^{-i p x} \mathrm{~d} x}{a^{2}+x^{2}}=\frac{\pi}{|a|} e^{-|a p|}
$$

for $a>0$ and $p$ real. Thus one gets

$$
\int_{-\infty}^{+\infty} \frac{e^{-i \epsilon_{\kappa} u} \mathrm{~d} u}{\left(12+u^{2}\right)}=\frac{\pi}{\sqrt{12}} e^{-\sqrt{12} \epsilon_{\kappa}}
$$

The final result is

$$
S_{4}=\frac{\kappa^{4} \epsilon_{\kappa}^{2}}{4 \pi^{2} \sqrt{12}} e^{-\sqrt{12} \epsilon_{\kappa}} .
$$

Interestingly, for a horizontal storage ring (guiding magnetic field in the vertical $z$ direction) the orbit in the moving frame can be approximated for laboratory times such that $\gamma \omega_{0}|t|=O(1)$ by the following semicubical parabola

$$
y^{\prime} \approx\left(\frac{R_{0}}{2 \gamma^{2}}\right)\left(\frac{6 \gamma^{2}\left|x^{\prime}\right|}{R_{0}}\right)^{2 / 3},
$$

where $R_{0}$ is the instantaneous radius of curvature of a particle's arbitrary trajectory.]

\footnotetext{
${ }^{1}$ See Fig. 2 and Eq. 2 in 22$]$.
} 


\section{The catenary.}

The worldline is $x^{\mu}(s)=\sigma^{-2}(\kappa \sinh \sigma s, \kappa \cosh \sigma s, \tau \sigma s, 0)$; with initial condition $x^{\mu}(0)=\sigma^{-2}(0, \kappa, 0,0)$. The integral is of the type

$$
\mathrm{I}_{5}=\sigma \int_{-\infty}^{+\infty} \frac{e^{-i \epsilon_{\sigma} u} \mathrm{~d} u}{2 \frac{\kappa^{2}}{\sigma^{2}}(\cosh u-1)-\frac{\tau^{2}}{\sigma^{2}} u^{2}},
$$

where $\frac{\tau^{2}}{\sigma^{2}}-\frac{\kappa^{2}}{\sigma^{2}}=-1$. This integral turns into $I_{2}$ and $I_{4}$ in the limits mentioned in the text, respectively, but again there is no helpful formula in the GR Table, and thus $I_{5}$ appears to be non-analytic.

\section{The helicoid (helix of variable pitch).}

This is the most general case. The worldline is

$$
\begin{aligned}
x^{\mu}(s)= & \left(\frac{\Delta}{R R_{+}} \sinh \left(R_{+} s\right), \frac{\Delta}{R R_{+}} \cosh \left(R_{+} s\right),\right. \\
& \left.\frac{\kappa \tau}{R \Delta R_{-}} \cos \left(R_{-} s\right), \frac{\kappa \tau}{R \Delta R_{-}} \sin \left(R_{-} s\right)\right) ;
\end{aligned}
$$

while the initial condition reads $x^{\mu}(0)=\left(0, \frac{\Delta}{R R_{+}}, \frac{\kappa \tau}{R \Delta R_{-}}, 0\right)$. I have defined:

$$
\begin{aligned}
\Delta^{2} & =\frac{1}{2}\left(R^{2}+\kappa^{2}+\tau^{2}+\nu^{2}\right) ; \\
R^{4} & =\left(\kappa^{2}+\tau^{2}+\nu^{2}\right)^{2}-4 \kappa^{2} \tau^{2} ; \\
R_{+}^{2} & =\frac{1}{2}\left(R^{2}+\kappa^{2}-\tau^{2}-\nu^{2}\right) ; \\
R_{-}^{2} & =\frac{1}{2}\left(R^{2}-\kappa^{2}+\tau^{2}+\nu^{2}\right) .
\end{aligned}
$$

The following integral is obtained:

$$
\mathrm{I}_{6}=R \int_{-\infty}^{+\infty} \frac{e^{-i \epsilon_{R} u} d u}{2\left(\frac{\Delta}{R_{+}}\right)^{2}\left[\cosh \left(\frac{R_{+}}{R} u\right)-1\right]+2\left(\frac{\kappa \tau}{\Delta R_{-}}\right)^{2}\left[\cos \left(\frac{R_{-}}{R} u\right)-1\right]} .
$$

This is the most complicated non-analytic stationary case, with no helpful formula in the GR Table.

\subsubsection{Non-stationary vacuum field noise}

Non-stationary vacuum field noise has a time-dependent spectral content requiring joint time and frequency information, i.e., one needs generalizations of the power spectrum concept. One can think of (i) tomographical processing and/or (ii) wavelet transforms. For instance, the recently proposed noncommutative tomography ( $\mathrm{NCT}$ ) transform $M(s ; \mu, \nu)$ [6] seems to be an attractive way of processing non-stationary signals. In the definition of $M, s$ is 
just an arbitrary curve in the non-commutative time-frequency plane, while $\mu$ and $\nu$ are parameters characterizing the curve. The most simple examples are the axes $s=\mu t+\nu \omega$, where $\mu$ and $\nu$ are linear combination parameters. The non-commutative tomography transform is related to the Wigner-Ville quasidistribution $W(t, \omega)$ by an invertible transformation and has the following useful properties

$$
\begin{aligned}
M(t ; 1,0) & =|f(t)|^{2} \\
M(\omega ; 0,1) & =|f(\omega)|^{2}
\end{aligned}
$$

where $f$ is the analytic signal which is simulated by $M$. Furthermore, employing $M$ leads to an enhanced detection of the presence of signals in noise which has a small signal-to-noise ratio. The latter property may be very useful in detecting VFNs, which are very small 'signals' with respect to more common noise sources.

On the other hand, since in the quantum detector method the vacuum autocorrelation functions are the essential physical quantities, and since according to various fluctuation-dissipation theorems they are related to the linear (equilibrium) response functions to an initial condition/vacuum, the fluctuationdissipation approach has been developed and promoted by Sciama and collaborators [7]. In principle, the generalization of the fluctuation-dissipation theorem for some classes of out of equilibrium relaxational systems, such as glasses, looks also promising for the case of non-stationary vacuum noise. One can use a so-called two-time fluctuation-dissipation ratio $X\left(t, t^{\prime}\right)$ and write a modified fluctuation-dissipation relationship [8]

$$
T_{\mathrm{eff}}\left(t, t^{\prime}\right) R\left(t, t^{\prime}\right)=X\left(t, t^{\prime}\right) \frac{\partial C\left(t, t^{\prime}\right)}{\partial t^{\prime}}
$$

where $R$ is the response function and $C$ the autocorrelation function. The fluctuation-dissipation ratio is employed to perform the separation of scales. Moreover, $T_{\text {eff }}$ are timescale-dependent quantities, making them promising for relativistic VFNs, which correspond naturally to out of equilibrium conditions.

\subsection{Circular electromagnetic vacuum noise}

\subsubsection{Introduction}

The circular electromagnetic vacuum noise, which in principle is more promising experimentally, 2 has been first discussed for specific purposes by Candelas and Deutsch, and by Bell and Leinaas. However, here we will pay more attention to the approach of Hacyan and Sarmiento (HS) [12 who in 1989 introduced a clear-cut and general method for calculating the main electromagnetic vacuum

\footnotetext{
${ }^{2}$ Rogers $[9]$ proposed to study the motion of a single electron in a Penning trap (geonium) to detect the circular electromagnetic vacuum noise. For two-level atoms in circular motion the reader is referred to Audretsch et al [1], whereas in the analog style approach Calogeracos and Volovik [1] considered the quasiparticle radiation from objects rotating in superfluid vacuum.
} 
spectral quantities and applied it to the basic cases of linear acceleration and uniform rotation. In the latter case, they obtained a nonzero energy flux in the direction of motion of the detector. It was this result that prompted Mane [13] to suggest a connection with the synchrotron radiation.

In principle, the circular vacuum noise power spectrum $S_{\mathrm{c}}$ could be calculated via the residue theorem, but the equation for the zeros of the denominator $x^{2}=v^{2} \sin ^{2} x$ (see below) is not analytically solvable. Nevertheless, for $v \geq 0.85$ one can expand the sine to find the zero with the smallest imaginary part, besides $x=0$ [14.

\subsubsection{The Hacyan-Sarmiento approach}

Starting with the expression for the electromagnetic energy-momentum tensor

$$
T_{\mu \nu}=\frac{1}{16 \pi}\left(4 F_{\mu \alpha} F_{\nu}^{\alpha}+\eta_{\mu \nu} F_{\lambda \beta} F^{\lambda \beta}\right) .
$$

Hacyan-Sarmiento define the electromagnetic two-point Wightman functions as follows

$$
\begin{gathered}
D_{\mu \nu}^{+}\left(x, x^{\prime}\right) \equiv \frac{1}{4}\left(4 F^{\alpha}{ }_{(\mu}(x) F_{\nu) \alpha}\left(x^{\prime}\right)+\eta_{\mu \nu} F_{\lambda \beta}(x) F^{\lambda \beta}\left(x^{\prime}\right)\right) \\
D_{\mu \nu}^{-}\left(x, x^{\prime}\right) \equiv D_{\mu \nu}^{+}\left(x^{\prime}, x\right) .
\end{gathered}
$$

This may be viewed as a variant of the "point-splitting" approach advocated by DeWitt. Moreover, because of the properties

$$
\eta^{\mu \nu} D_{\mu \nu}^{ \pm}=0, \quad D_{\mu \nu}^{ \pm}=D_{\nu \mu}^{ \pm}, \quad \partial_{\nu} D_{\mu}^{ \pm \nu}=0,
$$

the electromagnetic Wightman functions can be expressed in terms of the scalar Wightman functions as follows

$$
D_{\mu \nu}^{ \pm}\left(x, x^{\prime}\right)=c \partial_{\mu} \partial_{\nu} D^{ \pm}\left(x, x^{\prime}\right),
$$

where $c$ is in general a real constant depending on the case under study. This shows that from the standpoint of their vacuum fluctuations the scalar and the electromagnetic fields are not so different.

Now introduce sum and difference variables

$$
s=\frac{t+t^{\prime}}{2} ; \quad \sigma=\frac{t-t^{\prime}}{2} .
$$

Using the Fourier transforms of the Wightman functions

$$
\tilde{D}^{ \pm}(\omega, s)=\int_{-\infty}^{\infty} \mathrm{d} \sigma e^{-i \omega \sigma} D^{ \pm}(s, \sigma),
$$

where $\omega$ is the frequency of zero-point fields, the particle number density of the vacuum seen by the moving detector and the spectral vacuum energy density per mode are given by

$$
n(\omega, s)=\frac{1}{(2 \pi)^{2} \omega}\left[\tilde{D}^{+}(\omega, s)-\tilde{D}^{-}(\omega, s)\right],
$$




$$
\frac{\mathrm{d} e}{\mathrm{~d} \omega}=\frac{\omega^{2}}{\pi}\left[\tilde{D}^{+}(\omega, s)+\tilde{D}^{-}(\omega, s)\right] .
$$

The most important application of these results is to a uniformly rotating detector whose proper time is $s$ and angular speed is $\omega_{0}$ in motion along the circular world line

$$
x^{\alpha}(s)=\left(\gamma s, R_{0} \cos \left(\omega_{0} s\right), R_{0} \sin \left(\omega_{0} s\right), 0\right),
$$

where $R_{0}$ is the rotation radius in the inertial frame, $\gamma=\left(1-v^{2}\right)^{-1 / 2}$, and $v=\omega_{0} R_{0} / \gamma$. In this case there are two Killing vectors $k^{\alpha}=(1,0,0,0)$ and $m^{\alpha}(s)=\left(0,-R_{0} \sin \left(\omega_{0} s\right), R_{0} \cos \left(\omega_{0} s, 0\right)\right.$. Expressing the Wightman functions in terms of these two Killing vectors, HS calculated the following physically observable spectral quantities (i.e., those obtained after subtracting the inertial zero-point field contributions):

- The spectral energy density

$$
\frac{\mathrm{d} e}{\mathrm{~d} \omega}=\frac{\gamma^{3}}{2 \pi^{3} R_{0}^{3}} \frac{\omega^{2}+\left(\gamma v \omega_{0}\right)^{2}}{\omega^{2}} \frac{v^{3} w^{2}}{w^{2}+(2 \gamma v)^{2}} h_{\gamma}(w),
$$

- The spectral flux density

$$
\frac{\mathrm{d} p}{\mathrm{~d} \omega}=\frac{\gamma^{3}}{2 \pi^{3} R_{0}^{3}} \frac{\omega^{2}+\left(\gamma v \omega_{0}\right)^{2}}{\omega^{2}} 4 v^{4} k_{\gamma}(w),
$$

- The spectral stress density

$$
\frac{\mathrm{d} s}{\mathrm{~d} \omega}=\frac{\gamma^{3}}{2 \pi^{3} R_{0}^{3}} \frac{\omega^{2}+\left(\gamma v \omega_{0}\right)^{2}}{\omega^{2}} \frac{v^{3} w^{2}}{w^{2}+(2 \gamma v)^{2}} j_{\gamma}(w) .
$$

Here $\left(\omega^{2}+\left(\gamma v \omega_{0}\right)^{2}\right) / \omega^{2}$ is a density-of-states factor introduced for convenience and $h_{\gamma}(w), k_{\gamma}(w)$, and $j_{\gamma}(w)$ are the following cosine-Fourier transforms

$$
\begin{aligned}
& h_{\gamma}(w) \equiv \int_{0}^{\infty}\left(\frac{N_{h}(x, v)}{\gamma^{2}\left[x^{2}-v^{2} \sin ^{2} x\right]^{3}}-\frac{3}{x^{4}}+\frac{2 \gamma^{2} v^{2}}{x^{2}}\right) \cos (w x) \mathrm{d} x \\
& k_{\gamma}(w) \equiv-\int_{0}^{\infty}\left(\frac{N_{k}(x, v)}{\gamma^{2}\left[x^{2}-v^{2} \sin ^{2} x\right]^{3}}-\frac{3}{x^{4}}-\frac{\gamma^{2}}{6 x^{2}}\right) \cos (w x) \mathrm{d} x ; \\
& j_{\gamma}(w) \equiv \int_{0}^{\infty}\left(\frac{1}{\gamma^{4}\left[x^{2}-v^{2} \sin ^{2} x\right]^{2}}-\frac{1}{x^{4}}+\frac{2 \gamma^{2} v^{2}}{3 x^{2}}\right) \cos (w x) \mathrm{d} x .
\end{aligned}
$$

The numerators $N_{h}(x, v)$ and $N_{k}(x, v)$ are given by

$$
\begin{aligned}
& N_{h}(x, v)=\left(3+v^{2}\right) x^{2}+\left(v^{2}+3 v^{4}\right) \sin ^{2} x-8 v^{2} x \sin x \\
& N_{k}(x, v)=x^{2}+v^{2} \sin ^{2} x-\left(1+v^{2}\right) x \sin x .
\end{aligned}
$$

\footnotetext{
${ }^{3}$ This is correct for Galilean electromagnetism and works well at low velocities and/or in gradient index (lens) media. For full Lorentz covariant electrodynamics, one should use the Trocheris-Takeno nonlinear relationship $v=\tanh \left(\omega R_{0} / c\right)$. See, e.g., 15$]$.
} 
The employed variables are $w=\frac{2 \omega}{\omega_{0}}$ and $x=\frac{\sigma \omega_{0}}{2}$.

Of special interest are the ultra-relativistic and nonrelativistic limits. In the first case, $\gamma \gg 1$, the quantities

$$
H_{\gamma}=\frac{v^{3} w^{2}}{w^{2}+(2 \gamma v)^{2}} h_{\gamma}(w), \quad K_{\gamma}=4 v^{4} k_{\gamma}(w), \quad J_{\gamma}=\frac{v^{3} w^{2}}{w^{2}+(2 \gamma v)^{2}} j_{\gamma}(w),
$$

have the following scaling property

$$
X_{k \gamma}(k w)=k^{3} X_{\gamma}(w)
$$

where $k$ is an arbitrary constant, and $X=H, K, J$. This is the same scaling property as that of a Planckian distribution with a temperature proportional to $\gamma$.

A detailed discussion of the nonrelativistic limit has been provided by Kim, Soh, and Yee 16], who used the parameters $v$ and $\omega_{0}$, and not acceleration and speed as used by Letaw and Pfautsch for the circular scalar case 17]. They obtained a series expansion in velocity

$$
\frac{d e}{d \omega}=\frac{\omega^{3}}{\pi^{2}}\left[\frac{\omega_{0}}{\gamma \omega} \sum_{n=0}^{\infty} \frac{v^{2 n}}{2 n+1} \sum_{k=0}^{n}(-1)^{k} \frac{\left(n-k-\frac{\omega}{\gamma \omega_{0}}\right)^{2 n+1}}{k !(2 n-k) !} H\left(n-k-\frac{\omega}{\gamma \omega_{0}}\right)\right]
$$

where $H$ is the usual Heavyside step function. Thus, to a specified power of the velocity many vacuum harmonics could contribute; making the energy density spectrum quasi-continuous.

\subsubsection{Synchrotron radiation as electromagnetic vacuum fluctuations?}

In 1991, Mane used the Hacyan-Sarmiento formula for the energy flux to argue that its time component is related to the synchrotron radiation. The HacyanSarmiento Poynting flux is directed along the Lorentz boost from the laboratory frame to the rest frame of the observer, which is taken as the $y$ axis. It can be written

$$
p_{y}=\frac{1}{1440 \pi^{2}} \frac{\hbar \gamma^{8} \omega_{0}^{4} v}{c^{4}}\left(50-47 \gamma^{-2}\right)
$$

Note that $p_{y}$ is proportional to $\hbar$ and therefore becomes zero in the classical limit. However, for electrons which couple to this flux via the fine structure constant $\alpha=e^{2} / \hbar c$, the radiation effect looks totally classical. The recoil induced by the flux of the vacuum fluctuations on the four-momentum of the particle per unit proper time is

$$
\alpha A p_{y} \propto \frac{e^{2} \gamma^{4} \omega_{0}^{2} v}{c^{3}}
$$

where $A \approx R_{0}^{2} c^{2} /\left(v^{2} \gamma^{4}\right)$ is the transverse interaction area between the electron and the electromagnetic field. In the laboratory frame, the energy loss of the 
particle per unit laboratory time is given by the Larmor formula

$$
I=\frac{2}{3} \frac{e^{2}}{c^{3}}\left(\gamma^{2} \omega_{0} v\right)^{2}
$$

This is related to the damping force $\vec{F}$ in the form $I=\vec{F} \cdot \vec{v}$ and therefore the recoil induced by synchrotron radiation on the four-momentum of the particle per unit proper time is again proportional to $e^{2} \gamma^{4} \omega_{0}^{2} v / c^{3}$ as in Eq. (62). Therefore, the order of magnitude of the recoil of the particle induced by the $\alpha$ coupling to the vacuum flux is equal to that derived by the Larmor formula in the ultra-relativistic limit.

If one goes as far as accepting the idea that synchrotron radiation is due to noninertial electromagnetic vacuum fluctuations, one should reproduce in this approach all the many basic features of the synchrotron radiation that are known from both theory and measurements at storage rings. Recall, for example, that the Schwinger spectral intensity of the magneto-bremsstrahlung in the synchrotron regime [18] is proportional to the so-called shape function

$$
W_{\omega} \propto F\left(\frac{\omega}{\omega_{m}}\right)
$$

where $\omega_{m}$ is given in terms of the cyclotron radian frequency $\omega_{c}$ as $\omega_{m}=\omega_{c} \gamma^{3}$, and the shape function $F$ is given by

$$
F(\zeta)=\frac{9 \sqrt{3}}{8 \pi} \zeta \int_{\zeta}^{\infty} K_{5 / 3}(z) \mathrm{d} z,
$$

where $K$ is the MacDonald (modified Bessel) function of the quoted fractional order. The small and large asymptotic limits of the synchrotron shape function are as follows

$$
F(\zeta \ll 1) \approx 1.33 \zeta^{1 / 3}
$$

and

$$
F(\zeta \gg 1) \approx 0.78 \zeta^{1 / 2} e^{-\zeta},
$$

with a maximum (amount of radiation) to be found at the frequency $\omega_{m} / 3$. An examination of the Hacyan-Sarmiento asymptotic limits shows that there are clear differences between the Hacyan-Sarmiento and synchrotron energy density spectrum. Neither of the two limits coincide, neither the Hacyan-Sarmiento spectrum divided in two equal parts by its peak frequency as in the case of synchrotron radiation. Moreover, the well-defined polarization state of synchrotron radiation that can be calculated in closed form in terms of the squares of Bessel $K_{1 / 3}$ and $K_{2 / 3}$ functions in electrodynamics would prove really difficult to obtain in the vacuum approach. Finally, an expansion in velocity powers of the synchrotron radiation does not coincide with that in Eq. (60). In the opinion of the author, the circular electromagnetic vacuum noise should be considered as only a radiation signal embedded in the synchrotron radiation background. 


\subsubsection{Electron beam polarization at storage rings: Spin flip synchrotron radiation versus circular elec- tromagnetic vacuum noise}

Electromagnetic circular vacuum noise is interesting not only because of the Hacyan-Sarmiento results and Mane's suggestion but also as being responsible, according to Bell and Leinaas 19], for the electron depolarization at storage rings. This famous proposal was put under intense focus in 1998 at the Monterey conference organized by Pisin Chen, where one of the most authoritative contrarians, Professor J.D. Jackson declared [20:

Avoid the indiscriminate appeal to Unruh in order to "understand" something amenable to a simpler explanation.

The following is a brief introduction to this problem. It has been included here as an illustration of the confrontation of theoretical ideas with the experimental market; a confrontation eagerly awaited for the attractive analog proposals presented in this book.

The Bell-Leinaas proposal relies on the spin degree of freedom of the electron in an external magnetic field $B_{0}$ along the $z$ axis. The spin may be thought to have two (quasi)stationary states corresponding to $\sigma_{z}= \pm 1$, with an energy splitting $\Delta=2|\mu|\left|B_{0}\right|$. This approximation is valid when a second term in the effective spin-field interaction Hamiltonian, due to the so-called Thomas precession, is not included. Thus, the electron looks in a first approximation like an Unruh-DeWitt detector. The transitions between the two spin states induced by the radiation field are then written in terms of first-order timedependent perturbation theory, and a thermal ratio is obtained as if produced by the equilibrium ratio of populations of the upper and lower levels. The effect of the Thomas precession term in the effective Hamiltonian does not alter the shape of the polarization curve, and only shifts it horizontally when plotted as a function of the magnetic moment.

However, there is a simple quantum electrodynamical explanation of the polarization effect at storage rings in terms of the so called spin-flip synchrotron radiation that has been proposed by Sokolov and Ternov in 1963 [21. The spin-flip radiated power is very small with respect to the ordinary synchrotron radiation, becoming of the same order only at $\gamma_{s f}=\left(m c R_{0} / \hbar\right)^{1 / 2}$, which for a common storage ring is around $\gamma \approx 6 \times 10^{6}$. This is more than two orders of magnitude higher than the actual $\gamma<10^{4}$ of electrons in current storage rings leading to a spin-flip radiated power representing only $10^{-11}$ of the usual (non spin-flip) synchrotron emitted power. It is only because the spin flip accumulates over a time scale of tens of minutes to a few hours that one gets the observed asymptotic polarization $P_{l i m}=8 / 5 \sqrt{3}=0.924$.

It is either this non-stationarity of the spin-flip synchrotron radiation, or the fact that the orbiting electrons are actually more complicated interacting systems than simple Unruh-DeWitt detectors, that lead only to frequencydependent effective temperatures, which in the opinion of accelerator people are not useful parameters. 
The description of radiative polarization in terms of spin levels came under the scrutiny of Professor Jackson long ago [22]. He showed that the spacing between orbital levels is very small compared to the magnetic dipole M1 transition energy, and therefore the M1 transition will involve some changes in the orbital quantum number. In 1973, Derbenev and Kondratenko [23] obtained, in a quasi-classical approach, a formula for the equilibrium polarization in which spin-orbit effects are included through a spin-orbit coupling function. Their formula is considered to be the standard result for the transverse polarization at storage rings. According to the Derbenev-Kondratenko formula, for the range $0<g<1.2$ one of the levels is preferentially populated with respect to the other one. This effect cannot be reproduced in the Bell-Leinaas approach without resorting to time-dependent couplings and frequency-dependent 'temperatures' 14]. In 1987, Bell and Leinaas published a more detailed analysis of their proposal, in which (still assuming a thermal spectrum of the spin excitations) they took into account the fluctuations in the orbital motion. They obtained a polarization formula rather close to the standard one with some differences only close to a narrow depolarizing resonance. The claim is that when passing through the resonance the polarization falls from $92 \%$ to $-17 \%$ followed by an increase to $99 \%$ before settling again to $92 \%$.

Thus, the confirmation of their calculation, and of the thermal vacuum bath, as opposed to the Sokolov-Ternov limiting polarization would require precise experimental measurements of a transient passing through a depolarizing resonance, an experiment that is still to be performed.

\subsection{Unruh effect versus anomalous Doppler ef- fect}

The concept of the anomalous Doppler effect (ADE) was introduced in classical electrodynamics by Frank in 1942 [26]. ADE refers to the waves emitted within the Cherenkov cone by a "superluminal" oscillator moving in a refractive medium. (Frank's example is an electric dipole harmonically oscillating at angular frequency $\Omega$.) By definition, these waves exhibit an anomalous Doppler shift in the sense that their frequencies (with respect to $\Omega$ ) have a negative Doppler directivity factor $D$ (see below) and are given by $\omega_{A D E}=\Omega / D$. In the quantum version of this phenomenon, as discussed by Frolov and Ginzburg, one uses the energy-momentum conservation law for massless Bose radiation from a superluminal two-level detector to get the energy formula (68) below.

As we have already mentioned in Section (0.2.1), when studied with the detector method the Unruh effect for a detector with internal degrees of freedom is in some ways very similar to this anomalous Doppler effect (ADE), since in both cases the quantum detector is radiating 'photons' while passing into the upper level and not on the lower one [3] (see Fig.11).

This is refrained in the well-known conclusion of Unruh and Wald [24] when they considered the uniformly accelerated quantum detector looked upon from 


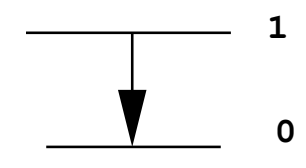

1
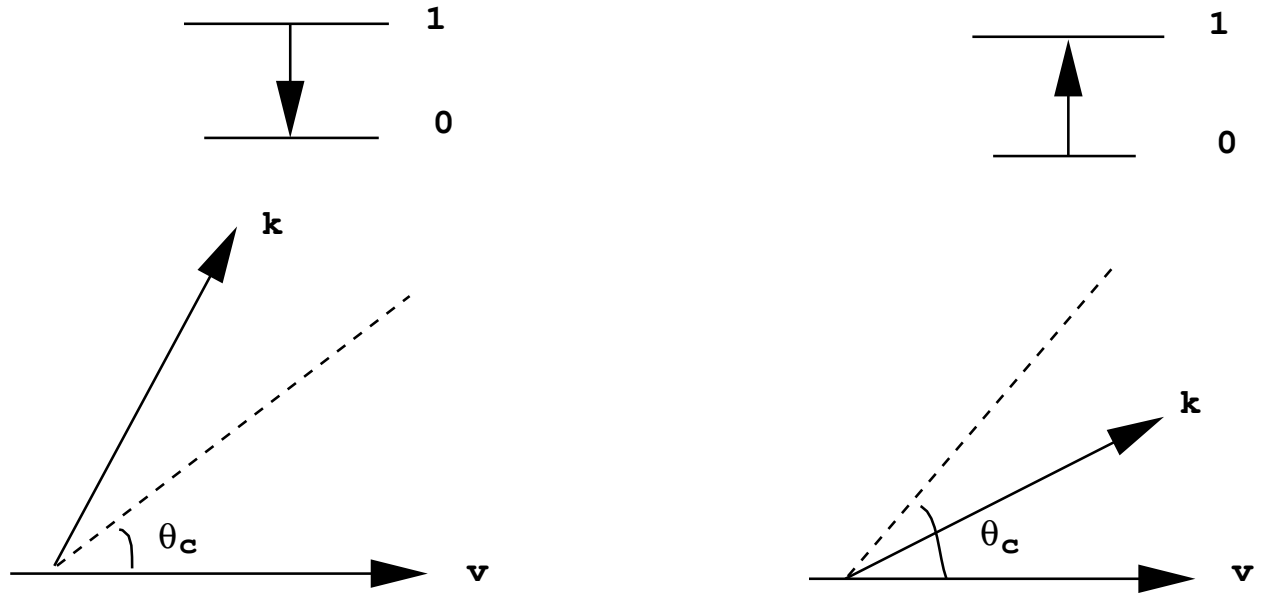

Figure 1: The normal and anomalous Doppler effects and the corresponding transitions.

the inertial reference frame:

When the observer places himself in an inertial reference frame then he is able to observe both the excited quantum detector (furnishing at the same time energy to it) and the 'photons'. By writing down the energy-momentum conservation law he will be inclined to say that the 'photons' are emitted precisely when the detector is excited.

Neglecting recoil, absorption, and dispersion (a completely ideal case) the elementary radiation events for a two-level detector with the change of the detector proper energy denoted by $\delta \epsilon$ are classified according to the photon energy formula [3]

$$
\hbar \omega=-\frac{\delta \epsilon}{D \gamma}
$$

where $\gamma$ is the relativistic velocity factor $(\gamma>1)$ and $\mathrm{D}$ is the Doppler directivity factor

$$
D=1-\left(\frac{v n}{c}\right) \cos \theta \text {. }
$$

The discussion of signs in Eq. (68) implies 3 cases as follows:

1. $\mathrm{D}>0$ for normal Doppler effect (NDE, $\delta \epsilon<0, \theta>\theta_{c}$ )

2. $\mathrm{D}=0$ for Cherenkov effect $\left(\mathrm{CE}, \delta \epsilon=0\right.$, undetermined case, $\theta=\theta_{c}$ )

3. $\mathrm{D}<0$ for anomalous Doppler effect $\left(\mathrm{ADE}, \delta \epsilon>0, \theta<\theta_{c}\right)$. 
Consequently, for a quantum system endowed with internal degrees of freedom the stationary population of levels is determined by the probability of radiation in the ADE and NDE regions. The possibility of inducing population inversion by means of the ADE has been extensively discussed in the literature since a long time ago 25].

Bolotovsky and Bykov [27] have studied the space-time properties of ADE in the simple case of a superluminal dipole $(v>c / n)$ propagating in uniform rectilinear motion in a non-dispersive medium. These authors claim a positive theoretical result with regard to the separate observation of the ADE phenomenon for this case. It is not, unfortunately, a realistic case and requires a special equation of motion of the dipole. Theoretical and experimental investigations of the possible manifestation of ADE in dispersive and/or lens media is an important task for the future.

A direct experimental evidence of $\mathrm{ADE}$ would be highly valuable as being equivalent to a test of the Unruh effect. The acoustic ADE is another challenge for the future [28.

\subsection{Summary}

As is the case for all claimed quantum vacuum effects (including the mechanical Casimir effect), the stationary radiative spectra surveyed in this chapter can be attributed equally well to radiation reaction fields, i.e., to solutions of the inhomogeneous Klein-Gordon or Maxwell equations evaluated at the source 29]. However, the main point we want to emphasize is different, namely: These radiation patterns (if they really exist - remember the calculations are all performed in first-order perturbation theory, or in order $\alpha$ for electrodynamic radiation reaction fields) can be used to extract radiometric information related to the Frenet-Serret geometric invariants of the trajectories of the relativistic corpuscules.

Finally, we emphasise that over the last few years the physics community has become aware of many interesting similarities/analogies between the Hawking/Unruh effects and shock-type effects in material media. A very promising line of research could be the study of the Cherenkov effect, and the associated anomalous and normal Doppler effects of relativistic dipoles propagating in strongly dispersive substances. Potentially realistic laboratory configurations for examining these effects are, for example, Cherenkov-type experiments with bunches of electric dipoles (polarization pulses) created by femtosecond optical pulses in electro-optic materials [30. In addition, radiation from vortices in two-dimensional annular Josephson junctions 31, or even from other 'relativistic' defects in condensed-matter physics should be taken into account from the perspective of this book.

Acknowledgements: The author would like to thank the organizers of the workshop for inviting him to this interesting event. 


\section{Bibliography}

[1] For review, see S. Takagi, "Vacuum noise and stress induced by uniform acceleration", Prog. Theor. Phys. Suppl. 88, 1 (1986). 0.2

[2] J.R. Letaw, "Stationary world lines and the vacuum excitation of noninertial detectors", Phys. Rev. D 23, 1709 (1981). 0.2

[3] V.P. Frolov and V.L. Ginzburg, "Excitation and radiation of an accelerated detector and anomalous Doppler effect", Phys. Lett. A 116, 423 (1986). $0.2 .1,0.4,0.4$

[4] W. Unruh, "Radiation reaction fields for an accelerated dipole for scalar and electromagnetic radiation", Phys. Rev. A 59, 131 (1999). 0.2.2

[5] E. Honig, E.L. Schucking, and C.V. Vishveshwara, "Motion of charged particles in homogeneous electromagnetic fields", J. Math. Phys. 15, 774 (1974).

For higher-dimensional generalization, see B.R. Iyer and C.V. Vishveshwara, "The Frenet-Serret formalism and black holes in higher dimensions", Class. Quant. Grav. 5, 961 (1988). 0.2 .2

[6] V.I. Man'ko and R. Vilela Mendes, "Noncommutative time-frequency tomography", Phys. Lett. A 263, 53 (1999) [physics/9712022]. D.2.4

[7] P. Candelas and D.W. Sciama, "Irreversible thermodynamics for black holes", Phys. Rev. Lett. 38, 1372 (1977). 0.2.4

[8] L.F. Cugliandolo, J. Kurchan and L. Peliti, "Energy flow, partial equilibration and effective temperatures in systems with slow dynamics", Phys. Rev. E 55, 3898 (1997) [cond-mat/9611044];

R. Exartier and L. Peliti, "Measuring effective temperatures in nonequilibrium systems", Eur. Phys. J. B 16, 119 (2000), [cond-mat/9910412]. 0.2 .4

[9] J. Rogers, "Detector for the temperature-like effect of acceleration", Phys. Rev. Lett. 61, 2113 (1988). 2

[10] J. Audretsch, R. Müller, and M. Holzmann, "Generalized Unruh effect and Lamb shift on arbitrary stationary trajectories", Class. Quant. Grav. 12, 2927 (1995). 2 
[11] A. Calogeracos and G.E. Volovik, "Rotational quantum friction in superfluids: Radiation from object rotating in superfluid vacuum", Pis'ma Zh. Eksp. Teor. Fiz. 69, 257 (1999).

[12] S. Hacyan and A. Sarmiento, "Vacuum stress-energy tensor of the electromagnetic field in rotating frames", Phys. Rev. D 40, 2641 (1989); Phys. Lett. B 179, 287 (1986). 2

[13] S.R. Mane, "Comment on HS", Phys. Rev. D 43, 3578 (1991);

See also, T. Hirayama and T. Hara, "A calculation on the self-field of a point charge and the Unruh effect", Prog. Theor. Phys. 103, 907 (2000) [gr-qc/9910111]. 目

[14] W.G. Unruh, "Acceleration radiation for orbiting electrons", Phys. Rept. 307, 163 (1998) [hep-th/9804158]. 2, 0.3.4

[15] S. Kichenassamy and R.A. Krikorian, "Note on Maxwell's equations in relativistically rotating frames", J. Math. Phys. 35, 5726 (1994);

R.D.M. De Paola and N.F. Svaiter, "A rotating vacuum and a quantum version of Newton's bucket experiment", Class. Quant. Grav. 18, 1799 (2001) [gr-qc/0009058]. 3

[16] S.K. Kim, K.S. Soh, and J.H. Yee, "Zero-point field in a circular-motion frame", Phys. Rev. D 35, 557 (1987). 3

[17] J.R. Letaw and J.D. Pfautsch, "Quantized scalar field in rotating coordinates", Phys. Rev. D 22, 1345 (1980). 3

[18] J. Schwinger, "On the classical radiation of accelerated electrons", Phys. Rev. 75, 1912 (1949). 0.3 .3

[19] J.S. Bell and J.M. Leinaas, "Electrons as accelerated thermometers", Nucl. Phys. B 212, 131 (1983); "The Unruh effect and quantum fluctuations of electrons in storage rings", Nucl. Phys. B 284, 488 (1987). 0.3.4

[20] J.D. Jackson, "On effective temperatures and electron spin polarization in storage rings", in Quantum aspects of beam physics, ed. Pisin Chen, World Scientific (1999) pp. 622-625 [physics/9901038]. 0.3.4

[21] A.A. Sokolov and I.M. Ternov, "On polarization and spin effects in the theory of synchrotron radiation", Dokl. Akad. Nauk 153, 1052 (1964) [Sov. Phys. Dokl. 8, 1203 (1964)]. 0.3.4

[22] J.D. Jackson, "On understanding spin-flip synchrotron radiation and the transverse polarization of electrons in storage rings", Rev. Mod. Phys. 48, 417 (1976).

For a recent review see, D.P. Barber, "Electron and proton spin polarization in storage rings - an introduction", in Quantum aspects of beam physics, ed. Pisin Chen, World Scientific (1999) pp. 67-90 [physics/9901038]. 且, 0.3 .4 
[23] Ya. S. Derbenev and A.M. Kondratenko, "Polarization kinetics of particles in storage rings", Zh. Eksp. Teor. Fiz. 64, 1918 (1973) [Sov. Phys. JETP 37, 968 (1973)]. 0.3 .4

[24] W.G. Unruh and R.M. Wald, "What happens when an accelerating observer detects a Rindler particle", Phys. Rev. D 29, 1047 (1984). 0.4

[25] See Section 11 and corresponding references in H. Rosu, "Hawking-like and Unruh-like effects: Toward experiments?", Gravitation \& Cosmology 7, 1 (2001) [gr-qc/9406012]. 0.4

[26] I.M. Frank, Izv. Akad. Nauk SSSR, Ser. Fiz. 6, 3 (1942). 0.4

[27] B.M. Bolotovski and V.P. Bykov, "On the theory of ADE", Radiofizika 32, 386 (1989). 0.4

[28] M.E. Goldstein, Aeroacoustics, (McGraw-Hill, New York, 1976). 0.4

[29] A.O. Barut and J.P. Dowling, "Quantum electrodynamics based on self fields: On the origin of thermal radiation detected by an accelerated observer", Phys. Rev. A 41, 2277 (1990). 0.5

[30] D.H. Austin, K.P. Cheung, J.A. Valdmanis, and D.A. Kleinman, "Cherenkov radiation from femtosecond optical pulses in electro-optic media", Phys. Rev. Lett. 53, 1555 (1984).

See also, T.E. Stevens, J.K. Wahlstrand, J. Kuhl, R. Merlin, "Cherenkov radiation at speeds below the light threshold: Phonon-assisted phase matching", Science 291, 627 (2001) and references therein. 0.5

[31] See, e.g., V.V. Kurin, A.V. Yulin, I.A. Shereshevskii, and N.K. Vdovicheva, "Cherenkov radiation of vortices in a two-dimensional annular Josephson junction", Phys. Rev. Lett. 80, 3372 (1998). 0.5 